\title{
Delineating Non-Covalent Interactions Between the Azinomycins and Double Stranded DNA: Importance of the Naphthalene Substitution Pattern on Interstrand Cross-Linking Efficiency
}

Cyrille A.S. Landreau, Rachel C. LePla, Michael Shipman,* Alexandra M.Z. Slawin, and John A. Hartley

Department of Chemistry, University of Warwick, Gibbet Hill Road, Coventry, CV4 7AL, U.K.

School of Chemistry, University of St. Andrews, Purdie Building, St. Andrews, Fife, KY16 9ST, U.K.

CR-UK Drug-DNA Interactions Research Group, Department of Oncology, Royal Free \& University College Medical School, University College London, 91 Riding House

Street, London WIW 7BS, U.K.

\section{Supporting Information}



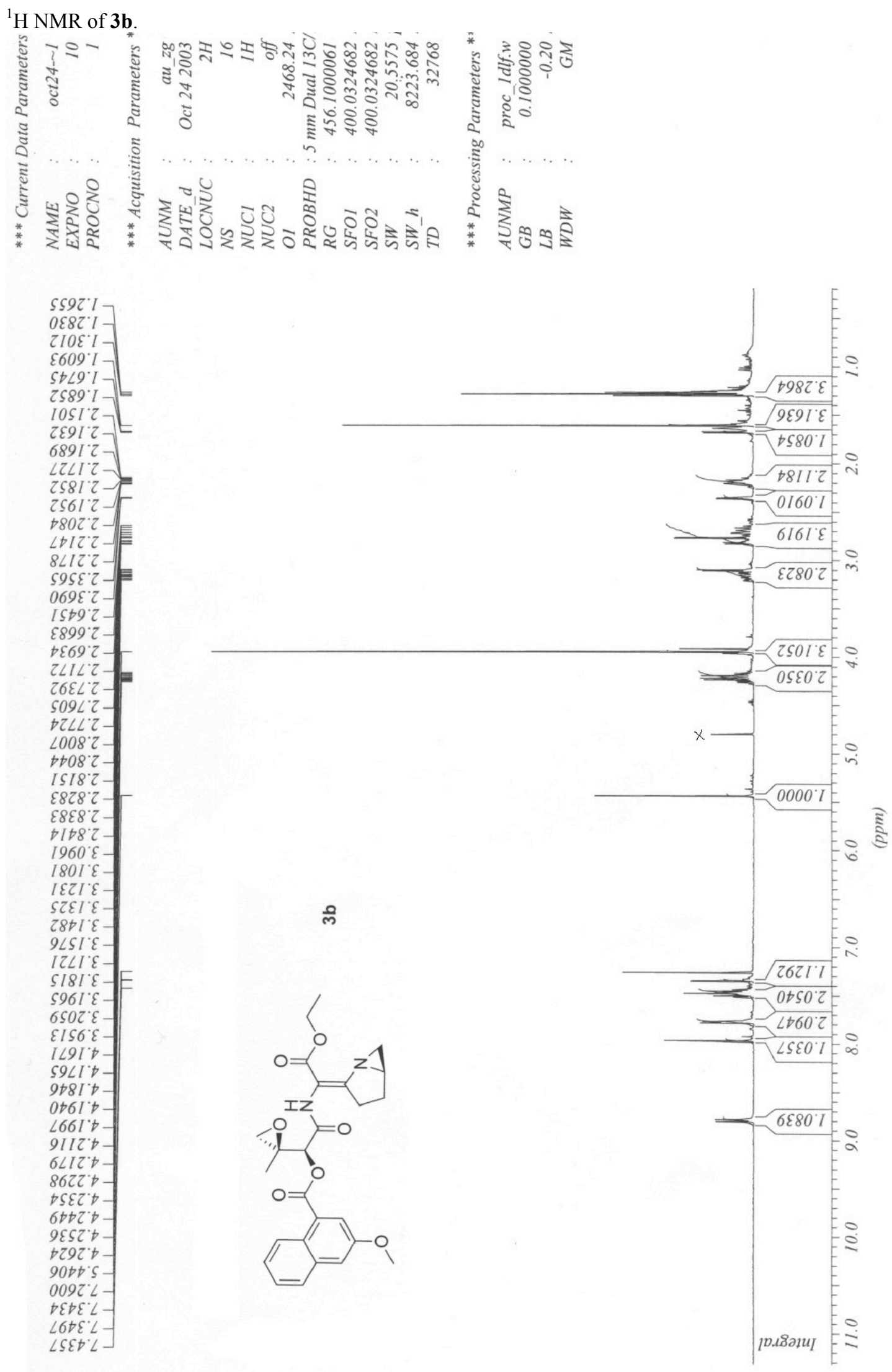

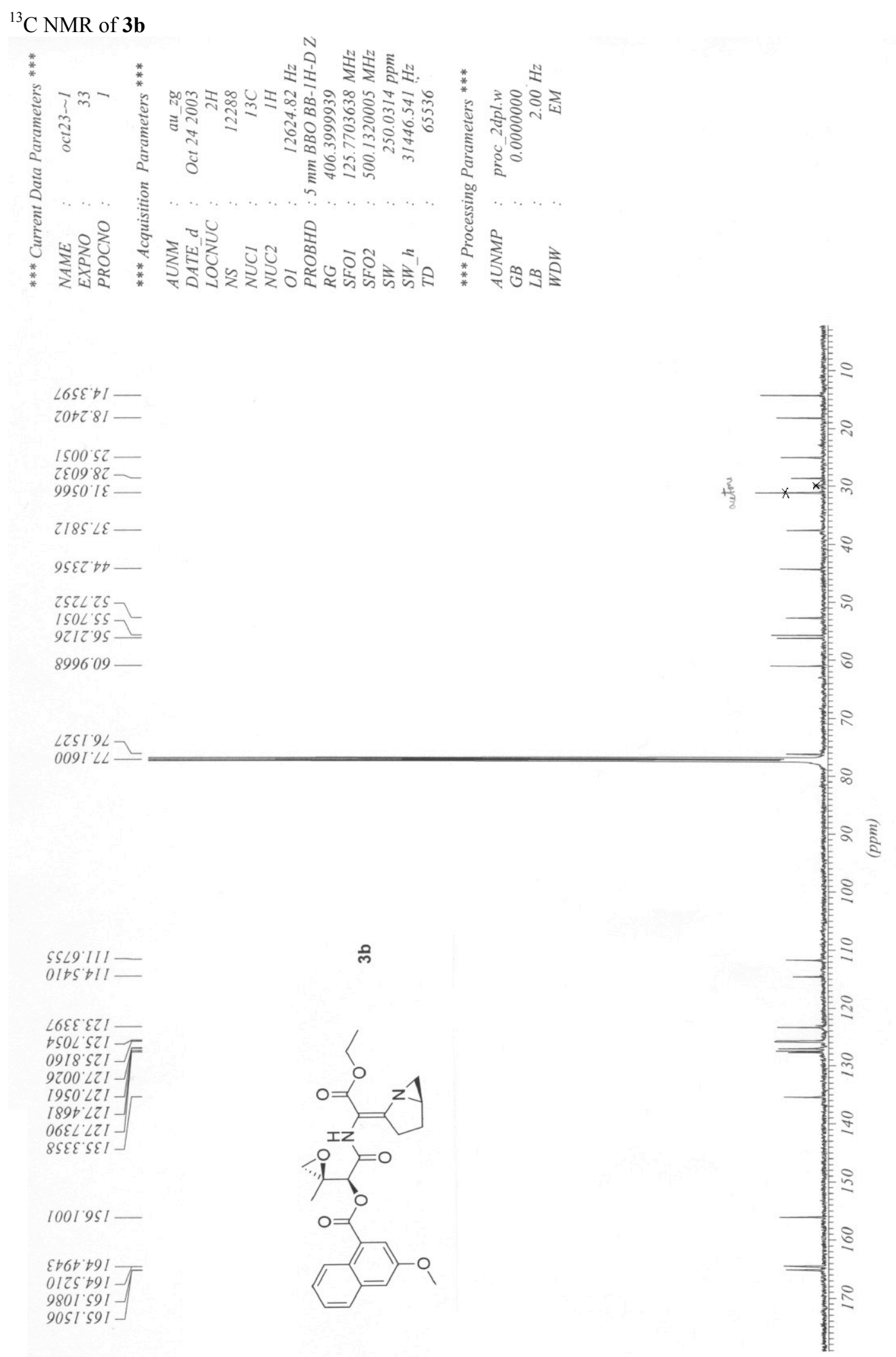


\section{${ }^{1} \mathrm{H}$ NMR of $\mathbf{3 c}$}

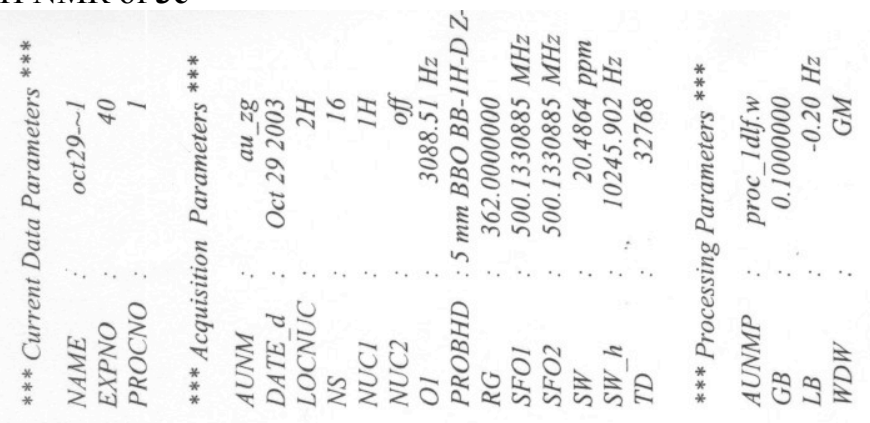

IE9 $I$

$S \angle L Z Z^{\circ} I$

61621

$8 \mathcal{E} 19^{\circ} \mathrm{I}$

$92297-$

$+89 I^{\circ} \mathrm{Z}-$

$96 \angle I ' Z$

$I \angle 8 I^{\prime} Z$

$066 I^{\prime} \mathrm{Z}$

$s 90 z \cdot 2$

$\tau \angle \mathcal{E E} Z>$

sE99.

$S 899^{\circ} \mathrm{Z}$
$\angle 189^{\circ} \mathrm{Z}$

$\angle 10 L ' \mathrm{C}$

$982 \angle ' Z$

$0 \mathcal{E} L L Z$

EZ8L'Z

$268 L^{\prime} \mathrm{Z}$

$9862 \mathrm{Z}-$

$9808^{\circ} \mathrm{Z}$

$2618^{\circ} \mathrm{Z}$

$6+I I \mathcal{E}-$

$\varepsilon \triangleright \tau I^{\circ} \varepsilon-$

$8 I t I^{\circ} \mathcal{E}-$

$\varepsilon \sigma t I^{\circ} \mathcal{E}$

$\varsigma 29 I^{\circ} \mathcal{E}$

$\varepsilon 691 \%$

$908 I^{\circ} \mathcal{E}-$

$I^{\prime 88 I^{\circ} \mathcal{E}-1}$

$\angle 80{ }^{\circ} \mathcal{E}$

$789 l^{\circ} \mathrm{t}$

$6 S L I$ '

8281 't

E06 I't

$066 I^{\circ} t$

$\angle t 02 \cdot t$

$t \varepsilon I Z^{\prime} t$

$06 I \tau^{\circ} t$

$8 \angle 2 Z^{\circ} t$

$\angle t \in Z^{\prime} t$

$z z+2 \cdot t$

$06+2 \circ-$

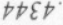

$0092: \angle$

SLLE

EI6E 2

EI6 ${ }^{\circ} \mathrm{L}$

$s+6 t^{\circ} \mathrm{L}$

$8805^{\circ} \mathrm{L}$

$2825^{\circ} \mathrm{L}$

$92+S^{\circ} L$

ists 2

S6SS $L$

$9 \angle 2 L L$

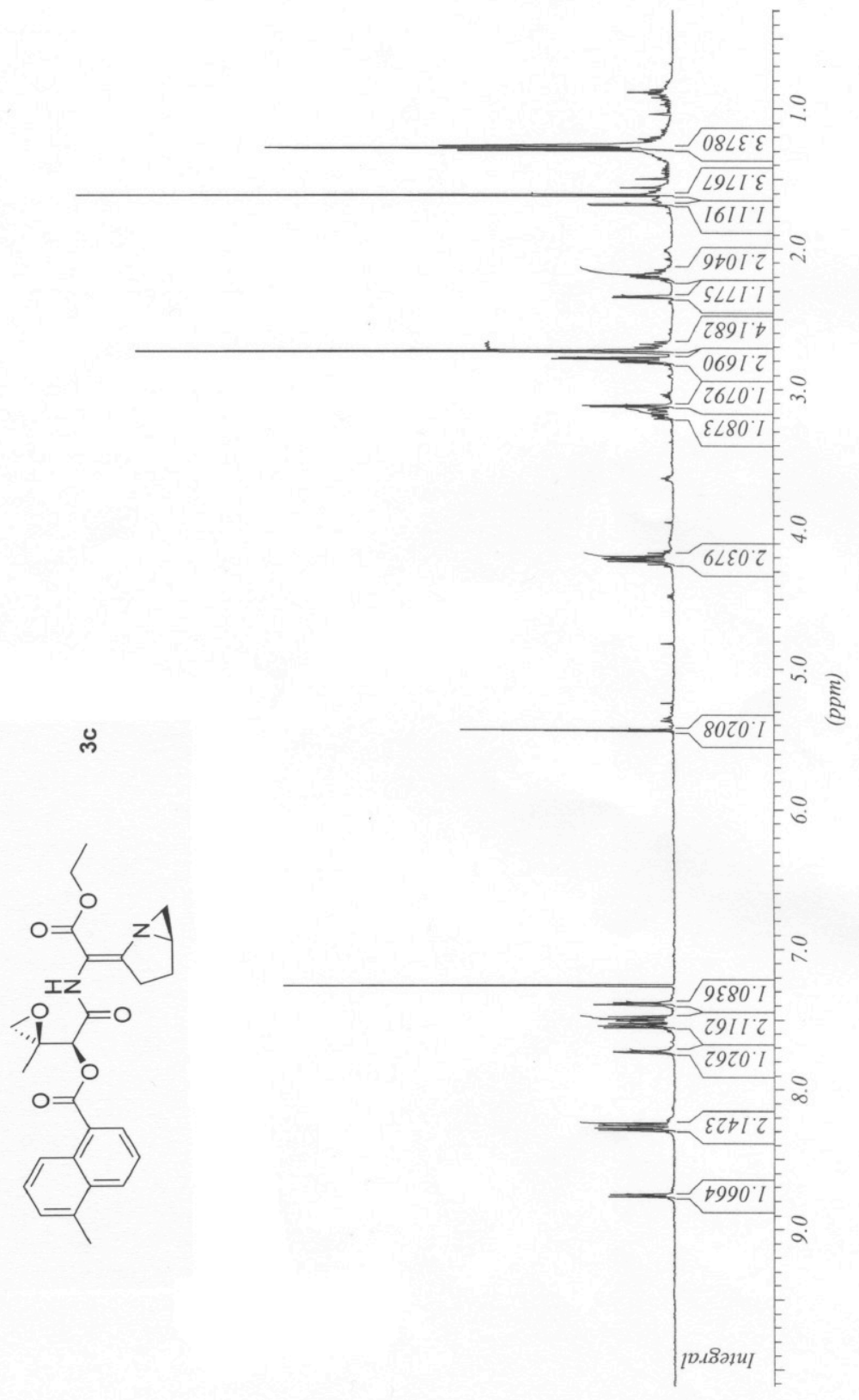




\section{${ }^{13} \mathrm{C}$ NMR of $\mathbf{3 c}$}

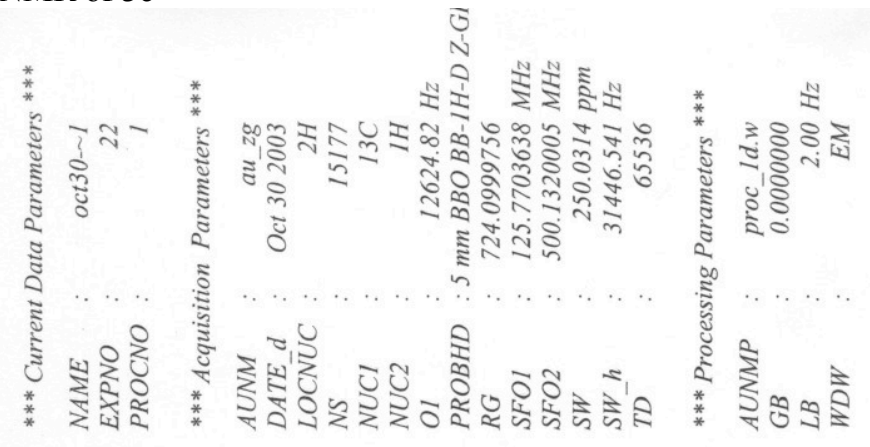

$978 \varepsilon \circ 1$

$020281-$

$6+00^{\circ} 02$

$9910^{\circ} \mathrm{SZ}$

$S 28 \angle L^{\circ} 82$

$\angle L \mathcal{E} L \angle \mathcal{E}$

0682 th

$\angle 006$ '

s962.9s

90<6.09

$\angle 6+0.94$

$0091 \% \mathrm{LL}$

m

O8Eカ・カI -

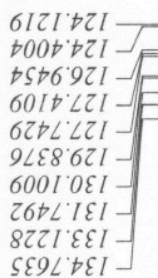

$\left.\begin{array}{l}90 \angle S+79 I- \\ t S+8.79 I \\ \angle D I E .59 I \\ 60 \angle 6.59 I\end{array}\right]$
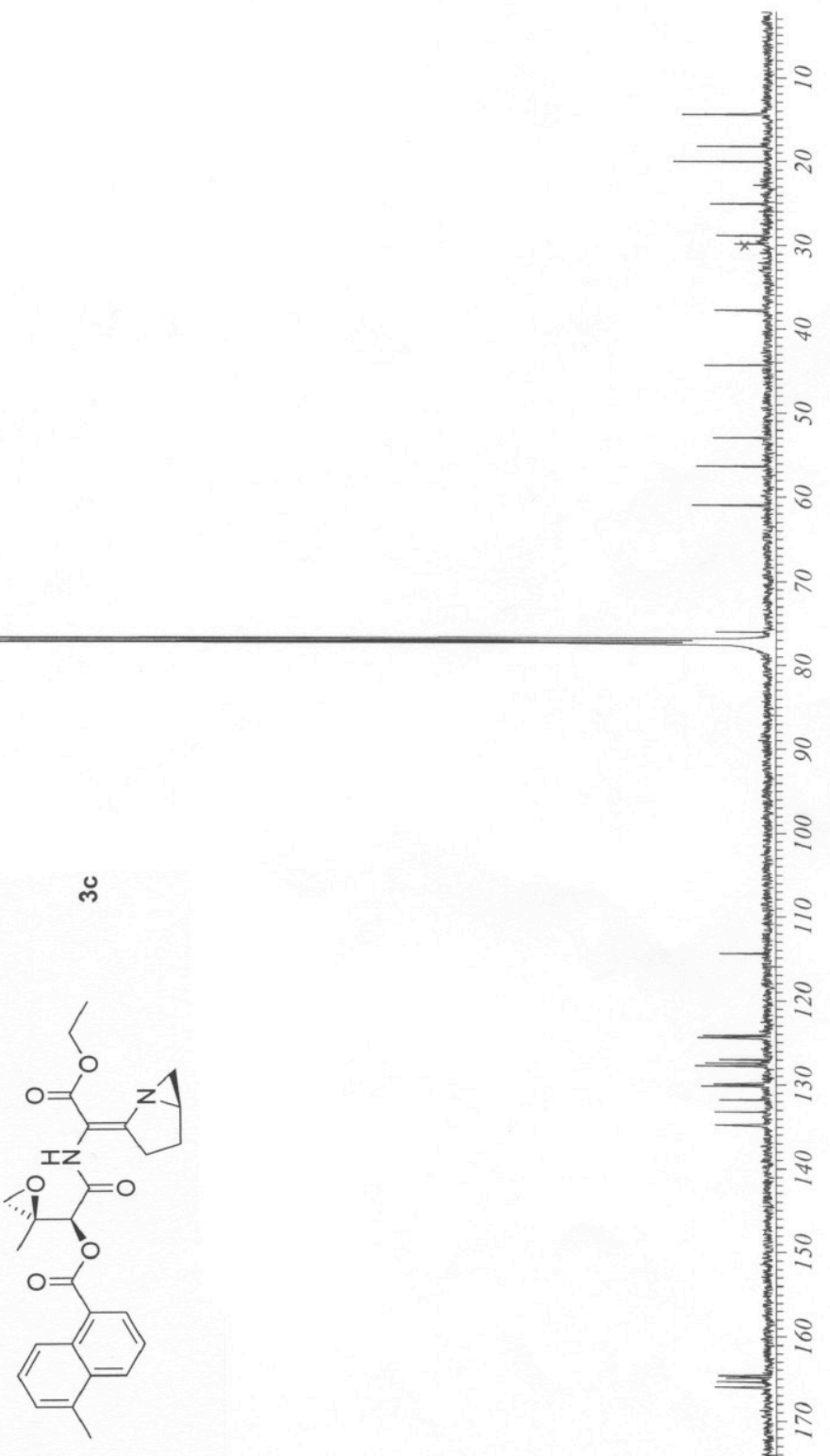

非 2 彥

\&

翡ミ 


\section{${ }^{1} \mathrm{H}$ NMR of $\mathbf{3 d}$}
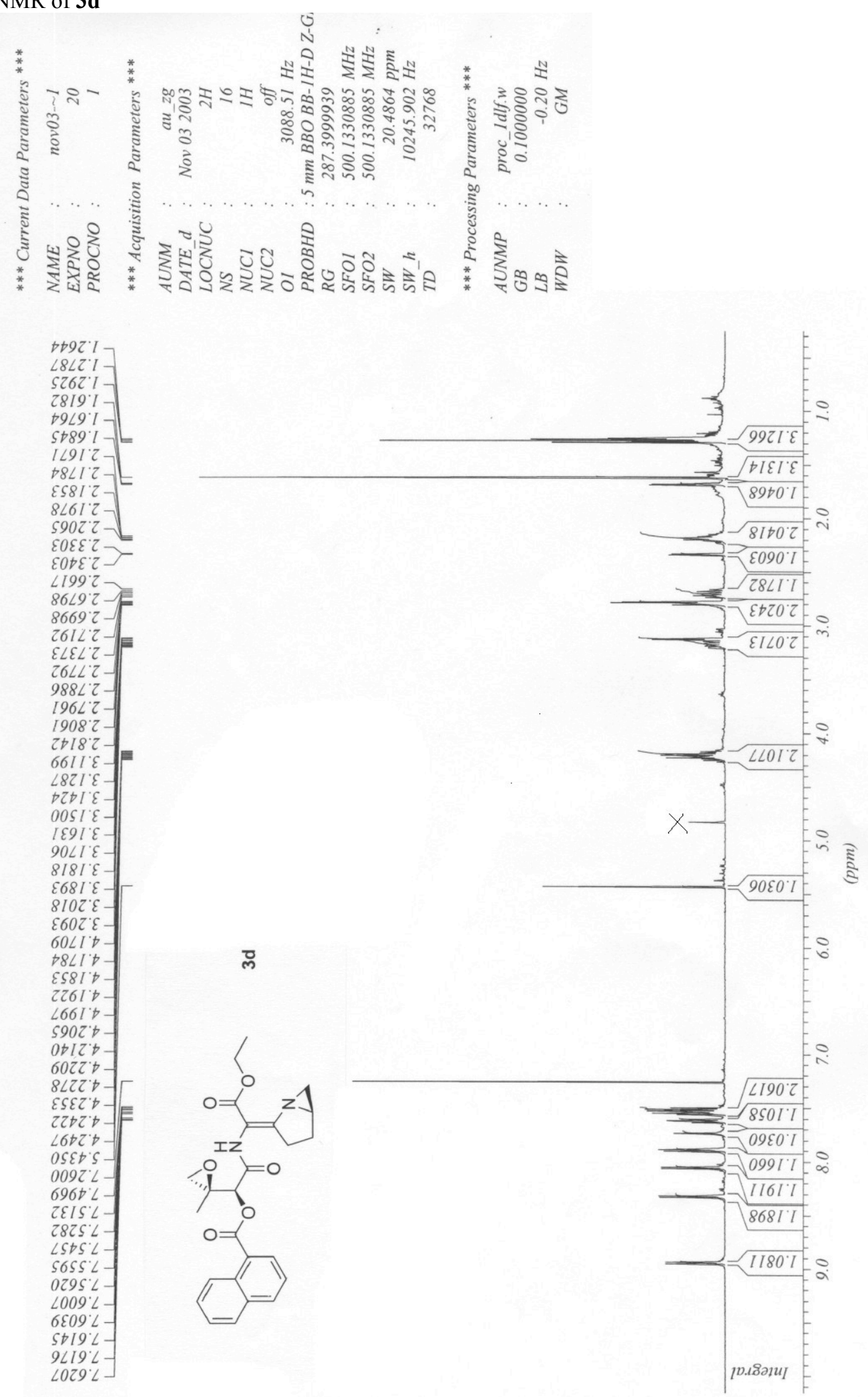


\section{${ }^{13} \mathrm{C}$ NMR of $\mathbf{3 d}$}

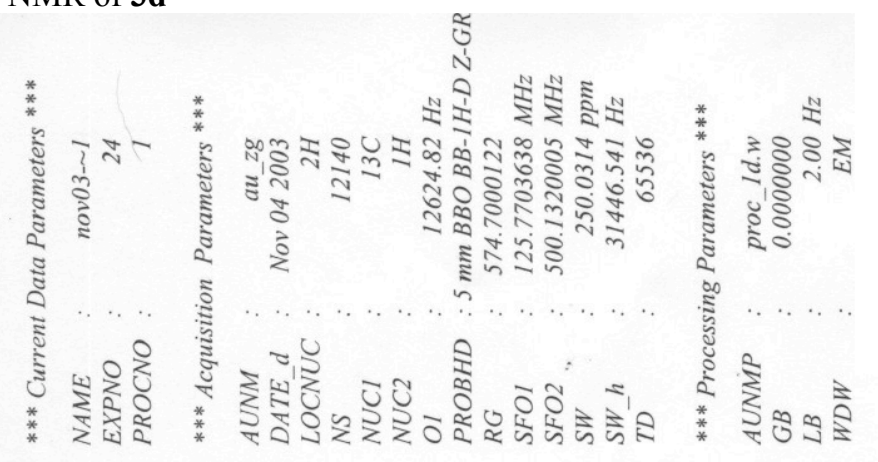

$88 \angle \mathcal{E} D I-$

ESLI'8I-

$6800^{\circ} \mathrm{SZ}$

$6+\angle L 82$

$8 \mathcal{E} \mathcal{L} L \mathcal{E}$

$8262 \cdot t t$

It56.25

6882.95

$++\angle 6.09$

$1190^{\circ} 94-$

$009 I^{\circ} \mathrm{LL}$
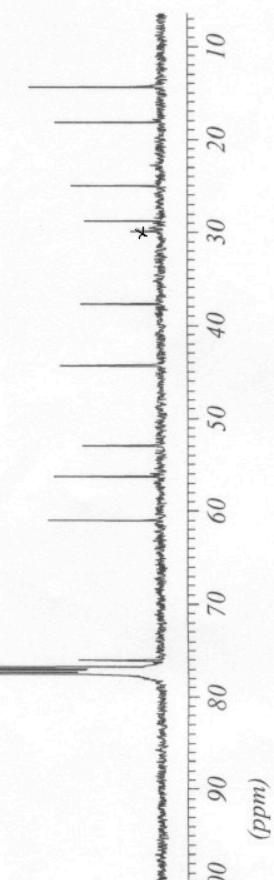

oses $t 9 I$

$\left.\begin{array}{l}0882.591 \\ \text { E978. }\end{array}\right]$

ల్ల

08Et7II
E0I9 $721-$

9L06'SZI

9SSI 921$]$

ILES $921-$

$9291821-$
$6269821-$

008LOEI

EEZ9. $[\mathcal{E} I]$

$\left.I \mathcal{E} \dagger \sigma^{\circ} \mathcal{E} \mathcal{E} I\right]$
$I 0 \mathcal{E}[\circ \mathcal{E} I]$

$0189^{\circ} 591$
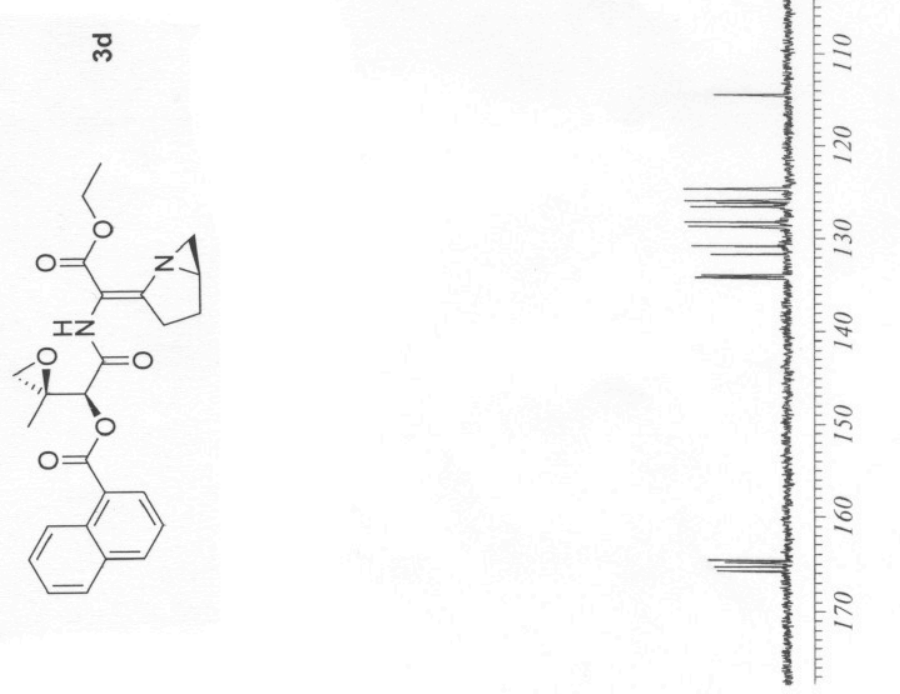